\title{
Aporte de la radiología oral y maxilofacial al diagnóstico clínico
}

\section{Contribution of oral and maxillofacial radiology to the clinical diagnosis}

\author{
Paz Gallardo C*, Celis Contreras C**, Schilling Quezada A**, \\ Schilling Lara $\mathrm{J}^{* *}$, Hidalgo Rivas $\mathrm{A} * * *$
}

\section{RESUMEN}

Introducción: La radiología oral y maxilofacial (RMF) es la disciplina encargada del uso de rayos $\mathrm{X}$ y otros tipos de radiación para el diagnóstico de enfermedades y condiciones del área maxilofacial. El propósito de la presente revisión es analizar el aporte de la RMF al diagnóstico clínico.

Revisión: Las legislaciones nacionales suelen establecer requisitos para autorizar el uso e instalación de equipos generadores de radiaciones ionizantes, sin embargo, falta regulación sobre la responsabilidad del diagnóstico radiográfico. Por esto, es necesario aplicar consideraciones éticas y principios de protección radiológica que garanticen el uso responsable de los exámenes radiográficos. Debiera existir una persona responsable del diagnóstico radiográfico, que debe ser un odontólogo con conocimientos en interpretación radiográfica y cursos de educación continua en RMF. La solicitud y toma radiográfica son acciones fundamentales que contribuyen a que el responsable del diagnóstico radiográfico realice uno correcto. La RMF actualmente es reconocida como especialidad en más de 50 países, sin embargo, existe la necesidad de reconocimiento en otros países. Debido a su formación profesional, la existencia de especialistas en RMF contribuye a mejores diagnósticos radiográficos, con el consecuente impacto en el diagnóstico clínico y tratamiento del paciente.

Conclusiones: La RMF es una disciplina fundamental para determinar el diagnóstico clínico del paciente. Para que los exámenes radiográficos usados aporten al clínico tratante a brindar la mejor atención al paciente, es fundamental que estos exámenes cuenten con un correcto y completo diagnóstico realizado por un profesional con entrenamiento y conocimientos en RMF, idealmente especialista en RMF

PALABRAS CLAVE: Odontología, Radiología, Imagenología Diagnóstica, Derivación y Consulta.

\section{ABSTRACT}

Introduction: Oral and maxillofacial radiology (MFR) is the discipline that deals with the use of X-rays and other kinds of radiation for the diagnosis of diseases and conditions of the maxillofacial region. The aim of the present

* Programa de Especialización en Imagenología Oral y Maxilofacial, Escuela de Graduados, Universidad de Talca, Chile.

** Programa de Especialización en Imagenología Oral y Maxilofacial, Escuela de Graduados, Universidad de Talca, Chile. Cirujano Dentista, Especialista en Radiología Oral y Maxilofacial, Departamento de Estomatología, Escuela de Odontología, Facultad de Ciencias de la Salud, Universidad de Talca, Chile.

*** Programa de Especialización en Imagenología Oral y Maxilofacial, Escuela de Graduados, Universidad de Talca, Chile. Cirujano Dentista, Especialista en Radiología Oral y Maxilofacial, Departamento de Estomatología, Escuela de Odontología, Facultad de Ciencias de la Salud, Universidad de Talca, Chile. Doctor en Radiología Oral. 
review is to analyze the contribution of MFR to the clinical diagnosis.

Review: National laws usually establish the requirements to authorize the use and installation of ionizing radiation generating equipment, nevertheless, there is a lack of regulation regarding the responsibility of the radiographic diagnosis. Because of this, it is necessary to apply ethical considerations and principles of radiation protection to guarantee the responsible use of the radiographic examinations. There should be a person responsible of the radiographic diagnosis, who must be a dentist with knowledge in radiographic interpretation and with continuing education courses in MFR. The radiographic request and taking are fundamental actions that contribute the responsible of radiographic diagnosis to elaborates a correct diagnosis. Nowadays, MFR is recognized as a specialty in more than 50 countries, however, there is a need of its acknowledgment in other countries. Due to the MFR specialist training, his/her existence contributes to a better radiographic diagnosis, with the consequent impact on clinical diagnosis and patient treatment.

Conclusions: MFR is a fundamental discipline for determining the clinical diagnosis of the patient. In order the radiographic exams contribute the treating clinician to offer the best clinical attention to the patient, it is fundamental these exams have a correct and complete radiographic diagnosis, performed by a professional with training and knowledge in MFR, ideally an MFR specialist.

KEY WORDS: Dentistry, Radiology, Diagnosis, Diagnostic Imaging, Referral and Consultation.

Fecha de recepción: 27 de febrero de 2019.

Fecha de aceptación: 3 de marzo de 2019.

Paz Gallardo C, Celis Contreras C, Schilling Quezada A, Schilling Lara J, Hidalgo Rivas A. Aporte de la radiología oral y maxilofacial al diagnóstico clínico. 2019; 35, (2): 73-82

\section{INTRODUCCIÓN}

La radiología oral y maxilofacial $(\mathrm{RMF})$ es la disciplina encargada del uso de los rayos $\mathrm{X}$ y otros tipos de radiación, para el diagnóstico de enfermedades y condiciones del área maxilofacial ${ }^{(1)}$. La RMF cuenta con diferentes exámenes radiográficos que pueden ser indicados para distintas situaciones clínicas ${ }^{(2)}$. Los exámenes radiográficos usados en RMF permiten diagnosticar patologías y hallazgos, controlar la evolución de lesiones, elaborar el plan de tratamiento y controlar el tratamiento en el tiempo ${ }^{(3)}$.

La falta de conocimiento del profesional acerca del marco legal y consideraciones éticas de la RMF, puede implicar que el odontólogo que solicita, toma o diagnostica los exámenes radiográficos, realice algun error que podría tener consecuencias legales ${ }^{(1)}$. Una inadecuada solicitud radiográfica, toma o diagnóstico del examen radiográfico puede conducir a un mal diagnóstico radiográfico y posiblemente a un mal diagnóstico clínico. Si bien diversos artículos definen distintos aspectos de la $\operatorname{RMF}^{(4,5,6,7)}$, no se encontró artículos que consoliden el aporte de la RMF al diagnóstico clínico. El propósito de la presente revisión es analizar el aporte de la RMF al diagnóstico clínico.

\section{MARCO LEGAL Y CONSIDERACIONES ÉTICAS DE LA RADIOLOGÍA ORAL Y MAXILOFACIAL}

Los exámenes radiográficos son documentos con valor médico-legal ${ }^{(1,8)}$. Debe controlarse su uso, ya que implican exponer al paciente a radiaciones ionizantes, que pese sus bajas dosis en odontología, suponen riesgo de producir efectos adversos en la salud del paciente y del personal ocupacionalmente expuesto (POE) (9). Estos efectos en odontología suelen ser estocásticos (probabilísticos), es decir, su aparición no depende de la dosis de radiación, sino del azar ${ }^{(9)}$. Debido a estos posibles efectos adversos, es fundamental que los odontólogos que realizan y utilizan los exámenes radiográficos, conozcan el marco legal y consideraciones éticas de la RMF. Las legislaciones nacionales suelen establecer requisitos para autorizar el uso e instalación de equipos generadores de radiaciones ionizantes. Estos requisitos varían de acuerdo al país ${ }^{(10,}$ $11,12)$, pero por lo general incluyen requisitos sobre aspectos comunes, tales como: a) equipo generador de radiaciones ionizantes, b) lugar físico de la instalación, c) titular de la instalación, d) medidas de protección radiológica, e) operador (persona que realiza el examen) y f) personal que se desempeña en la instalación. Organismos internacionales, como la Comisión 
Europea y la Academia Americana de Radiología Oral y Maxilofacial, recomiendan la necesidad del diagnóstico radiográfico de exámenes de la región maxilofacial $^{(3,13,14)}$. De hecho, se describe que se corre el riesgo de perder una demanda por negligencia profesional debido a la falta de diagnóstico radiográfico ${ }^{(15,16)}$. Sin embargo, las legislaciones nacionales suelen no referirse a la responsabilidad del diagnóstico radiográfico.

Considerando la falta de regulación legal sobre la responsabilidad del diagnóstico radiográfico, es necesario que el odontólogo que realiza y usa los exámenes radiográficos aplique ciertas consideraciones éticas fundamentales. Esto, con la finalidad de garantizar el uso responsable, seguro y efectivo de los exámenes radiográficos ${ }^{(17)}$. La Comisión Internacional de Protección Radiológica (ICRP, del inglés International Commission on Radiological Protection) promueve la aplicación de valores éticos fundamentales ${ }^{(18)}$ : a) Beneficencia y no maleficencia: procurar el mayor beneficio en salud y nunca hacer daño, b) Prudencia: tomar decisiones cuidadosa y razonablemente, c) Justicia: distribución equitativa de ventajas y desventajas en el bienestar de la persona, y d) Dignidad: respeto incondicional a los derechos humanos y autonomía de las personas. Estos valores éticos se relacionan con los tres principios de protección radiológica establecidos por la ICRP. El principio de justificación implica valorar que la toma del examen radiográfico genere más beneficio que riesgo para el paciente ${ }^{(18)}$. El principio de optimización significa obtener el examen con la menor exposición a radiación posible para el paciente, sin perder información diagnóstica ${ }^{(18)}$. Los principios de justificación y optimización se relacionan directamente con el paciente. El principio de limitación de dosis se aplica al POE, el cual establece mantener los niveles de exposición ocupacional a radiación por debajo de los límites de dosis máximos permitidos. El principio de justificación se relaciona con los valores éticos de beneficencia, no maleficencia y prudencia ${ }^{(18)}$. El principio de optimización se relaciona con prudencia, justicia y dignidad ${ }^{(18)}$. El principio de limitación de dosis se relaciona con prudencia y justicia ${ }^{(18)}$. Los valores éticos fundamentales integrados con los principios de protección radiológica contribuyen a que el odontólogo que realiza y usa los exámenes radiográficos actúe adecuadamente al proteger al paciente de las radiaciones ionizantes ${ }^{(18)}$.

Desde el punto de vista ético, la ficha clínica del paciente es un documento privado y confidencial ${ }^{(19)}$, así como la información y exámenes clínicos y radiográficos contenidos en ella ${ }^{(1)}$. Esto significa que toda persona que utilice la ficha clínica tiene la obligación de guardar secreto sobre la misma ${ }^{(19)}$. Adicionalmente, es fundamental que exista una persona responsable del diagnóstico radiográfico una vez obtenido el examen ${ }^{(20)}$.

\section{Diagnóstico RADIOGRÁFICO EN ODONTOLOGía}

El diagnóstico del examen radiográfico es esencial en el proceso de diagnóstico de enfermedades ${ }^{(1)}$, al no haberlo, se arriesga perder información importante para la determinación del diagnóstico clínico. El responsable del diagnóstico radiográfico debe ser un odontólogo con conocimientos en interpretación radiográfica ${ }^{(1,21)}$ y cursos de educación continua en el área de la $\mathrm{RMF}^{(3)}$ que lo capaciten para interpretar el examen completo. Además, este odontólogo debe mostrar confiabilidad y certeza en lo que describe, empleando las palabras adecuadas para dar a conocer el diagnóstico radiográfico ${ }^{(20)}$.

La solicitud y la toma radiográfica son acciones fundamentales, que contribuyen a que el responsable del diagnóstico radiográfico realice un diagnóstico correcto. Por esta razón, el clínico tratante que solicita el examen, el operador y el responsable del diagnóstico deben conocer las responsabilidades que les competen. En general, estas personas tienen la responsabilidad de aplicar los principios de protección radiológica antes, durante y después de la toma radiográfica ${ }^{(1)}$. El clínico tratante que solicita el examen radiográfico tiene la responsabilidad de conocer el rendimiento de cada examen, para solicitar el que más contribuya a determinar el diagnóstico clínico ${ }^{(3,13,22)}$. Además, debe comunicarse adecuadamente con el responsable del diagnóstico radiográfico. Esta comunicación por parte del clínico tratante se produce mediante la solicitud radiográfica, la cual debe ser completada con los datos completos del paciente, información clínica, e indicando el propósito del examen radiográfico ${ }^{(23)}$. Esto contribuye a que el responsable del diagnóstico radiográfico responda la pregunta clínica y realice un diagnóstico con mayor precisión ${ }^{(23)}$.

El operador tiene la responsabilidad de aplicar los conocimientos necesarios y poseer la habilidad para realizar correctamente la toma radiográfica y el procesado radiográfico ${ }^{(1)}$. Tras la toma y procesado radiográfico, el operador debe realizar el control de calidad de la imagen ${ }^{(24,25)}$. Esto permite garantizar una imagen radiográfica de calidad para su correcta interpretación por parte del responsable del diagnóstico. 
Una vez obtenido el examen radiográfico, el responsable del diagnóstico radiográfico tiene la responsabilidad de realizar el diagnóstico y enviarlo al clínico tratante que lo solicita. Mediante esta acción, se produce la adecuada comunicación entre el responsable del diagnóstico y el clínico tratante que solicita el examen radiográfico. El responsable del diagnóstico debe asegurar las adecuadas condiciones de visualización de los exámenes radiográficos, tales como: a) iluminación ambiental de la sala de diagnóstico ${ }^{(24,26)}$ y b) condiciones de observación para exámenes radiográficos convencionales y digitales, como por ejemplo características del negatoscopio o especificaciones técnicas del monitor ${ }^{(24)}$. Las adecuadas condiciones de visualización contribuyen a la elaboración de un correcto diagnóstico radiográfico. Adicionalmente, el responsable del diagnóstico radiográfico debe realizar el diagnóstico del examen siendo consciente que éste puede repercutir en el tratamiento del paciente ${ }^{(27)}$ y su planificación ${ }^{(28)}$. Debido a esto, el responsable del diagnóstico radiográfico debe analizar el examen radiográfico de forma sistemática $^{(2,29)}$, es decir, mediante una evaluación secuencial y ordenada, específica para cada tipo de examen radiográfico ${ }^{(2)}$. Una evaluación sistemática, así como la revisión del diagnóstico radiográfico una vez finalizado, permiten evitar errores en el diagnóstico. Los errores en el diagnóstico radiográfico pueden referirse a: a) error en la transcripción de la información, b) mal uso de terminología, c) omisión de alguna lesión o hallazgo o d) diagnóstico ambiguo poco preciso que no transmite claramente la información ${ }^{(30)}$. Adicionalmente, el responsable del diagnóstico debe tener conocimiento de una serie de aspectos que le permitan realizar un diagnóstico correcto, tales como: a) formación de imagen, b) anatomía, c) lesiones y d) hallazgos de la región maxilofacial.

a) Formación de imagen: estos aspectos permiten determinar si la imagen radiográfica, convencional o digital, es apta para el diagnóstico radiográfico. En exámenes radiográficos convencionales, debe evaluarse que cuenten con la correcta densidad (grado de oscurecimiento del negativo radiográfico), contraste (diferencias en las densidades) y características geométricas de nitidez (precisión de las líneas que componen la imagen), magnificación (imagen con mayor tamaño que el real del objeto radiografiado) y distorsión (imagen alterada en el tamaño y forma real del objeto radiografiado) ${ }^{(1)}$. La figura 1 muestra una

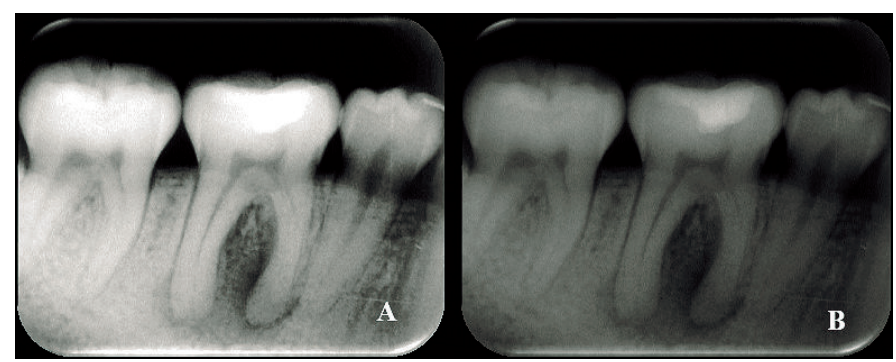

Figura 1. A) Radiografía con baja densidad. B) Radiografía con la correcta densidad, apta para el diagnóstico.

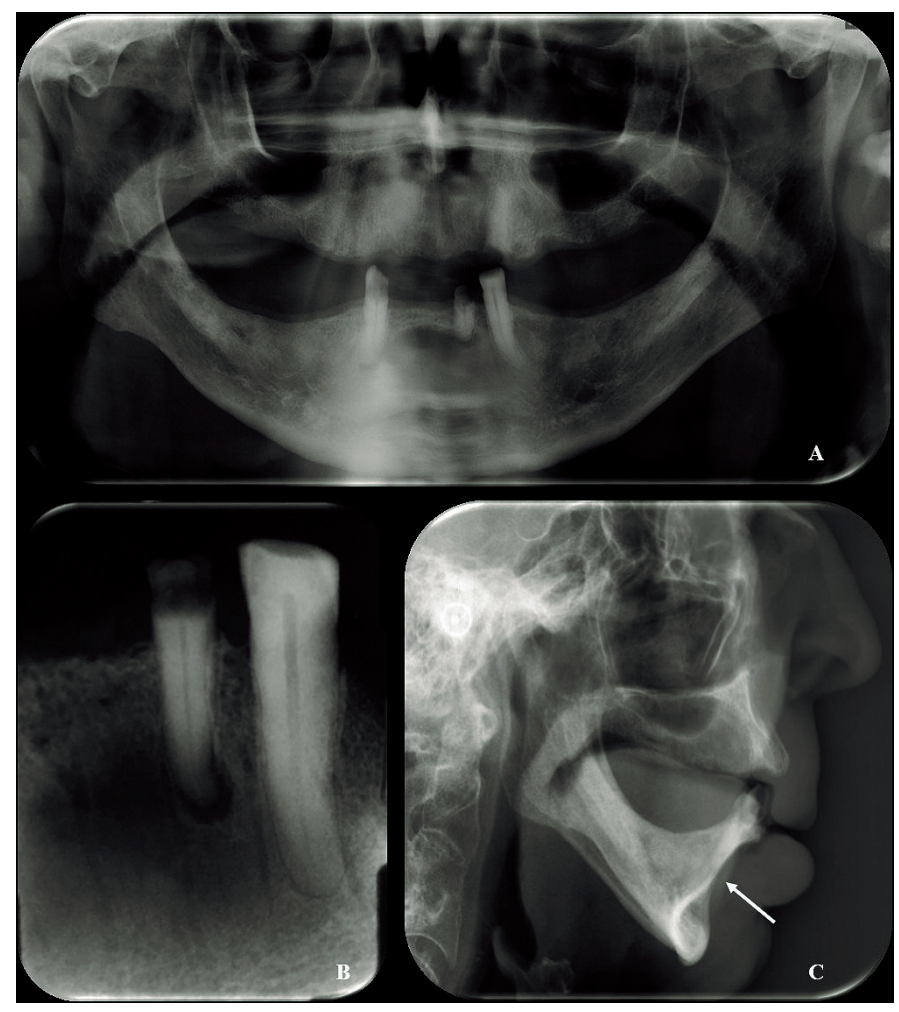

Figura 2. Paciente de 76 años. A) En radiografía panorámica se observa imagen radiolúcida de forma ovalada de contorno regular, parcialmente corticalizada en relación con el ápice del diente 3.2, que se extiende hacia mesial del diente 4.3. El especialista en RMF complementa el estudio con radiografía periapical y telerradiografía de perfil. B) En radiografía periapical se observa un granuloma apical en diente 3.2 y un trabeculado óseo de aspecto difuso. C) En sección de telerradiografía de perfil se observa una tabla ósea vestibular muy adelgazada a nivel de la zona sinfisiaria de la mandíbula y el proceso alveolar anteroinferior. Se descarta presencia de patología y, por consiguiente, la necesidad de una intervención quirúrgica. Conclusión diagnóstica: Colapso de tabla ósea vestibular (variación anatómica).

radiografía con baja densidad y otra con la correcta densidad.

En exámenes radiográficos digitales, el responsable del diagnóstico debe considerar elementos 
adicionales, tales como ruido y artefactos. El ruido se produce por las diferencias de atenuación del haz de rayos $X$, que producen diferencias de captación de la radiación por los pixeles de la imagen digital, resultando en pixeles más oscuros y otros más claros ${ }^{(31)}$. El grado de ruido depende de aspectos tales como: a) cantidad de la radiación ionizante usada, es decir, del miliamperaje $(31)$, b) características técnicas del detector o c) procesado de la imagen digital ${ }^{(13)}$. Con relación a los artefactos, estos elementos son producidos por efectos absorcionales ${ }^{(32)}$, se visualizan en el examen radiográfico digital sin estar presentes en el objeto de estudio, y pueden dificultar el diagnóstico radiográfico de la imagen obtenida ${ }^{(5)}$.

b) Anatomía: el diagnóstico radiográfico correcto de las estructuras anatómicas evita complicaciones durante y/o después del tratamiento ${ }^{(33)}$. El responsable del diagnóstico radiográfico debe conocer la anatomía dentaria y de estructuras adyacentes que se observan en el examen completo (27, 34). Mientras más grande el área radiografiada, más son las estructuras anatómicas que evaluar ${ }^{(34)}$, lo que exige mayor conocimiento del responsable del diagnóstico radiográfico. Esto, debido a que pueden aparecer estructuras anatómicas de dominio poco habitual del odontólogo, como columna cervical o base de cráneo. El responsable del diagnóstico radiográfico debe reconocer las variaciones normales anatómicas, para distinguirlas de posibles patologías ${ }^{(5)}$. La figura 2 resalta la importancia del reconocimiento de la anatomía normal y sus variaciones.

c) Lesiones: son condiciones patológicas que afectan la región maxilofacial, que pueden ser benignas o malignas ${ }^{(2)}$. Para un correcto diagnóstico radiográfico, las lesiones deben ser observables en su totalidad en el examen radiográfico ${ }^{(2,27)}$. Además, la lesión debiera observarse al menos en dos planos del espacio para determinar sus características radiográficas: a) ubicación anatómica, b) límites/márgenes (netos corticalizados o no corticalizados, difusos o infiltrados), c) tamaño y extensión y d) relación con estructuras adyacentes (2). Sin embargo, es ideal observar la lesión en los tres planos del espacio, para establecer una mejor hipótesis diagnóstica y posible pronóstico del paciente. Ciertas características radiográficas, tales como límites netos corticalizados o expansión de corticales, hacen sospechar de una lesión benigna. Por otro lado, características radiográficas

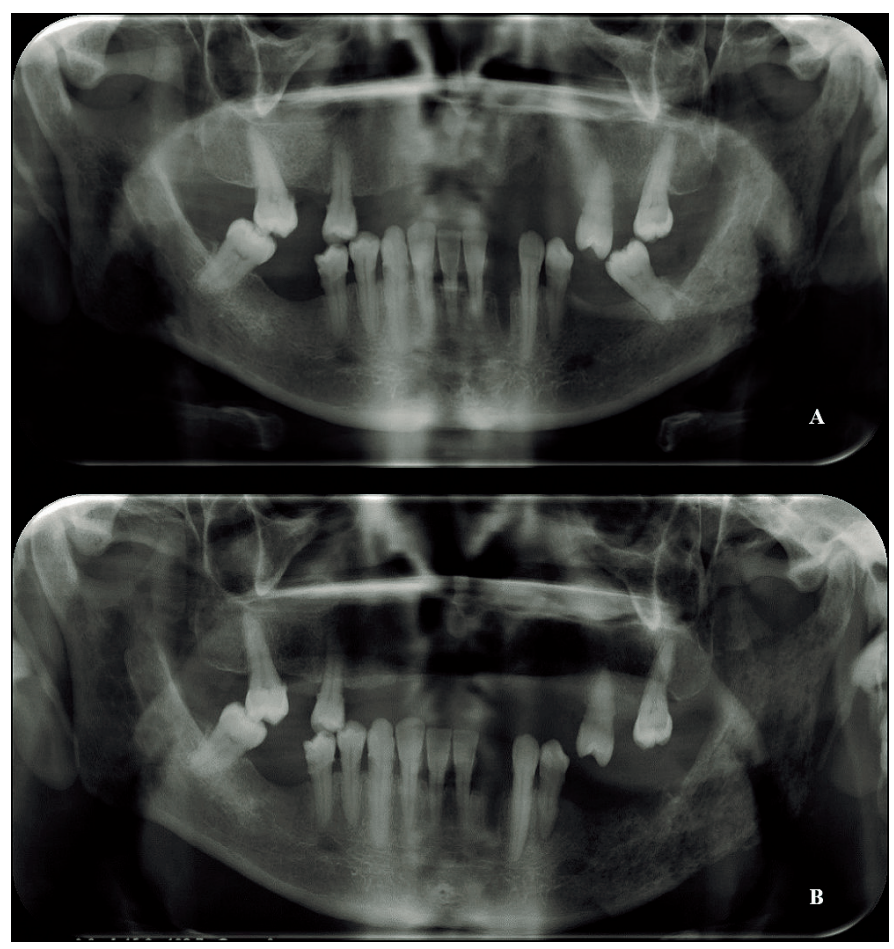

Figura 3. Paciente refería dolor en zona de diente 3.8; el clínico tratante realizó extracción del diente sin toma radiográfica previa. Poco tiempo después, persiste el dolor. A) El clínico tratante solicita radiografía panorámica, sin solicitar el diagnóstico radiográfico, y realiza extracción de diente 3.7 . Tres meses después, la paciente se continúa quejando de dolor en la zona mandibular izquierda. B) El clínico tratante solicitó nuevamente una radiografía panorámica, esta vez con su correspondiente diagnóstico radiográfico. El especialista en RMF diagnosticó una alteración del trabeculado óseo de la zona mandibular izquierda, de bordes mal definidos y adelgazamiento de la cortical a nivel del ángulo mandibular.

Conclusión diagnóstica: Metástasis de adenocarcinoma de mama.

como límites difusos, infiltración ósea e invasión a espacios anatómicos adyacentes, hacen sospechar de una lesión agresiva o incluso maligna ${ }^{(35)}$. El no determinar correctamente las características radiográficas puede llevar a un diagnóstico clínico erróneo, pudiendo implicar complicaciones en salud para el paciente. Por esto, el responsable del diagnóstico radiográfico debe identificar estas características a tiempo para que el clínico tratante realice oportunamente el tratamiento o derivación del paciente al especialista correspondiente ${ }^{(35)}$. La figura 3 muestra un caso en que el clínico realizó un diagnostico radiográfico erróneo, resultando en el fallecimiento de la paciente.

d) Hallazgos radiográficos: corresponde a la detección inesperada de una variante anatómica normal o una condición patológica, no relaciona- 


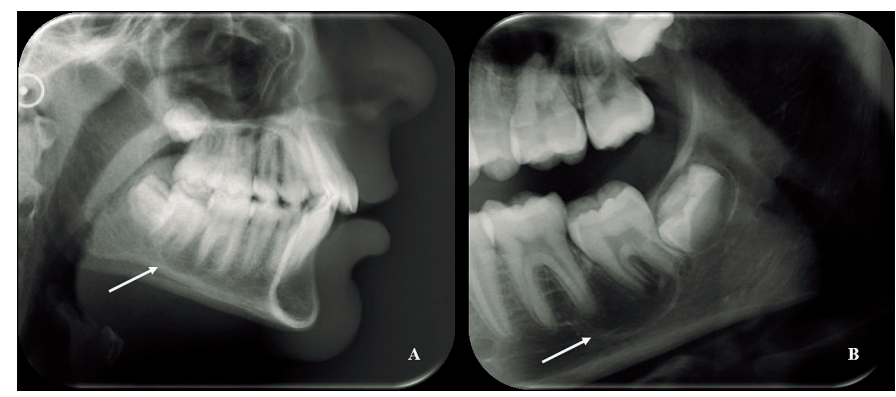

Figura 4. Paciente de 13 años que consultó por tratamiento ortodóntico. A) En sección de la telerradiografía de perfil se observa una lesión radiolúcida de forma redondeada aparentemente corticalizada, en relación con las raíces de los primeros y segundos molares inferiores. El especialista en RMF diagnosticó este hallazgo y complementó el estudio con radiografía panorámica. B) En sección de radiografía panorámica se observa la lesión en relación con el diente 3.7 en evolución extraósea y con leve inclinación vestibulolingual.

Conclusión diagnóstica: Quiste mandibular bucal infectado.

da con el propósito del examen ${ }^{(21)}$. La figura 4 muestra un hallazgo radiográfico en un paciente asintomático que consultó por tratamiento de ortodoncia. En la región maxilofacial, los hallazgos radiográficos pueden tener una alta prevalencia, que suele ser mayor en exámenes con tomografía computarizada de haz cónico ${ }^{(21)}$ que en exámenes bidimensionales (2D) ${ }^{(36,37)}$. Estos hallazgos pueden implicar que el clínico tratante realice distintas acciones: a) no realizar ningún tipo de intervención $^{(38)}$, b) realizar solamente un seguimiento $(39)$, c) complementar con otros exámenes para obtener un diagnóstico más preciso y determinar el tratamiento ${ }^{(40)}$ o d) realizar una intervención inmediata ${ }^{(41)}$. El clínico tratante podría no realizar la correcta acción si obtiene un diagnóstico radiográfico con errores. Debido a esto, el responsable del diagnóstico radiográfico debe estar consciente que incluso puede poner en riesgo la vida del paciente ${ }^{(42)}$.

\section{ROL DE LA RADIOLOGÍA ORAL Y MAXILOFACIAL}

En base a lo descrito, es posible afirmar que la RMF es fundamental como apoyo al clínico tratante para la determinación del diagnóstico del paciente. La RMF es reconocida como especialidad odontológica a la fecha en más de 50 países ${ }^{(43)}$, sin embargo, en algunos de estos países, este reconocimiento es reciente ${ }^{(44)}$. En Santiago de Chile, en 1968, se fundó la Asociación Internacional de Radiología DentoMaxiloFacial (IADMFR, del inglés International Association of DentoMaxilloFacial Radiology). Desde esa época, la IADMFR repre- senta a la RMF a nivel mundial, y establece entre sus propósitos apoyar al reconocimiento y desarrollo de la $\mathrm{RMF}^{(45)}$. Por otra parte, la Asociación Dental Americana establece requerimientos para el reconocimiento de una especialidad en Estados Unidos ${ }^{(6)}$. Algunos de estos requerimientos son: a) demostrar que es un área de la odontología que requiere conocimientos y habilidades que no poseen los odontólogos generales y demás especialistas, b) demostrar la necesidad y demanda de los servicios ofrecidos por la especialidad y que no han podido ser cumplidas por odontólogos generales y c) demostrar el beneficio directo del cuidado del paciente a través de la especialidad ${ }^{(6)}$. El cumplimiento de estos requerimientos permitió el reconocimiento de la $\mathrm{RMF}$ como especialidad en dicho país. Esto permite justificar la necesidad del reconocimiento de la especialidad de $\mathrm{RMF}$ en otros países, considerando los beneficios que aporta a la odontología.

En los países donde la RMF no es reconocida como especialidad, posiblemente no existen especialistas en RMF o, si existen, no hay suficientes para colaborar con todos los clínicos tratantes. Además, puede ocurrir que un especialista en RMF no sea valorado como especialista, dificultando su incorporación en universidades ${ }^{(46)}$ o centros clínicos multidisciplinarios. La inexistencia de especialistas en RMF o su falta de valoración, implica que el clínico tratante sería quien toma y/o diagnostica los exámenes radiográficos de sus pacientes ${ }^{(44)}$ y en otros casos, puede ser incluso el radiólogo médico quien diagnostique algunos exámenes radiográficos extraorales ${ }^{(44)}$. Se ha observado deficiencias de los odontólogos en: solicitud de exámenes radiográficos (47), identificación de la técnica intraoral utilizada (46), dosis de exposición a la radiación del paciente ${ }^{(48)}$, procesado de la imagen ${ }^{(48)}$ y diagnóstico radiográfico de lesiones ${ }^{(29,42)}$. Con respecto al diagnóstico radiográfi$\mathrm{co}$, se ha comparado el realizado por especialistas en RMF con el realizado por odontólogos generales $(49,50)$ y especialistas no radiólogos ${ }^{(29,51,52)}$ mostrando que el especialista en RMF diagnostica con mayor precisión $(29,49,50,51,52)$. Además, se ha observado que luego de un entrenamiento en diagnóstico radiográfico impartido por un especialista en RMF a odontólogos generales y especialistas no radiólogos, mejora su precisión en el diagnóstico, sin embargo, no logran diagnosticar la totalidad de las lesiones y/o hallazgos ${ }^{(51)}$.

Un odontólogo no entrenado o sin conocimientos en el área de la RMF, pudiera no estar familiarizado con regiones anatómicas alejadas de los dientes, por lo que es posible que no diagnostique lesiones presentes en el examen radiográfico ${ }^{(33)}$. Debido a esto, es necesa- 
ria la colaboración de un especialista en $\mathrm{RMF}$, quien cuenta con competencias prácticas y teóricas adquiridas durante su entrenamiento y ejercicio profesional $(30,53)$. Además, el especialista en RMF está familiarizado con la anatomía normal y sus variaciones ${ }^{(53)}$, por lo que se le facilita el reconocimiento y descripción de las mismas, así como la detección de cualquier lesión o hallazgo radiográfico. Además, el especialista en RMF está capacitado para hacerse cargo de un departamento de radiología en una universidad, hospital o centro privado ${ }^{(54)}$, asegurando el cumplimiento óptimo de sus funciones. Estas competencias permiten al especialista en $\mathrm{RMF}$ desempeñarse correctamente en el área de la $\mathrm{RMF}$ y diagnosticar exámenes radiográficos con propiedad, siendo capaz de alertar al clínico tratante sobre cualquier alteración que pueda pasarle inadvertida ${ }^{(29)}$.

Es recomendable que exista una buena comunicación entre odontólogos generales/especialistas no radiólogos y el especialista en $\mathrm{RMF}^{(55)}$. Esta comunicación contribuye a: a) seleccionar el examen radiográfico más adecuado ${ }^{(7)}$, b) proveer protección radiológica ${ }^{(56)}$, c) establecer parámetros de control de calidad de la imagen ${ }^{(57)}$, d) disminuir errores en el diagnóstico radiográfico $^{(27,58)}$ y, consecuentemente, e) disminuir errores en el tratamiento ${ }^{(58)}$. Una buena comunicación entre el clínico tratante y el especialista en RMF resulta en una mejor atención y un mayor beneficio para el paciente.

\section{CONSIDERACIONES FINALES}

La RMF es un apoyo al clínico tratante y aporta al beneficio del paciente. Pese a esto, es posible que en algunos países no se realice el diagnóstico radiográfico de la totalidad de exámenes. Esto puede deberse a la falta de regulación legal sobre la responsabilidad y obligatoriedad del diagnóstico radiográfico y/o a la falta de odontólogos capacitados para realizar el diagnóstico radiográfico. Es conveniente que los países incorporen en sus leyes esta regulación, considerando el impacto de un correcto diagnóstico radiográfico en el diagnóstico clínico. Además, para una mejor realización y uso del examen y diagnóstico radiográfico, es conveniente que aumente el número de países en que la RMF es reconocida como especialidad.

El odontólogo que se desempeñe en RMF debe tener conocimiento avanzado en múltiples aspectos para cumplir correctamente su función, especialmente en diagnóstico radiográfico ${ }^{(33,58,59)}$. Pese a que existen numerosas guías que orientan al odontólogo a un mejor desempeño en $\operatorname{RMF}^{(1,2,3,13,22,54)}$, es el especialista en
RMF quien cuenta con la formación profesional específica que le permite desempeñarse idóneamente en esta área.

En conclusión, la aplicación del marco legal y consideraciones éticas de la RMF por parte del odontólogo que realiza y usa los exámenes radiográficos, permite su uso responsable, brindando una atención eficiente y segura al paciente. Además, disminuye la posibilidad de demandas por negligencia profesional hacia el odontólogo. Para el correcto diagnóstico radiográfico es necesario que el clínico tratante que solicita el examen, el operador y el responsable del diagnóstico posean los conocimientos y habilidades necesarias para realizar correctamente su función. Además, debe existir una adecuada comunicación entre el clínico tratante que solicita el examen radiográfico y el responsable del diagnóstico radiográfico. La RMF es fundamental para contribuir en la determinación del diagnóstico clínico del paciente. Para que los exámenes radiográficos aporten al clínico tratante a brindar la mejor atención al paciente, es fundamental que estos exámenes cuenten con un correcto y completo diagnóstico realizado por un profesional con entrenamiento y conocimientos en RMF, idealmente especialista en RMF.

\section{BIBLIOGRAFIA}

1. Iannucci JM, Howerton LJ. Dental radiography principles and techniques. Elsevier/Saunders: Missouri; 2012.

2. Whaites E, Drage N. Essentials of dental radiography and radiology. Churchill Livingstone, Elsevier: Edinburgh; 2013.

3. European Commission. Radiation protection No 136. European guidelines on radiation protection in dental radiology: the safe use of radiographs in dental practice. 2004.

4. Kamburoglu K. Dento-maxillofacial radiology as a specialty. World J Radiol 2015; 7:87-8.

5. Husain MA. Dental anatomy and nomenclature for the radiologist. Radiol Clin North Am 2018; 56:111.

6. Scarfe WC. Oral and maxillofacial radiology as a dental specialty: the first decade. Oral Surg Oral Med Oral Pathol Oral Radiol Endod 2010; 110:4058. 
7. Vandenberghe B, Jacobs R, Bosmans H. Modern dental imaging: a review of the current technology and clinical applications in dental practice. Eur Radiol 2010; 20:2637-55.

8. SOCIÉTÉ FRANÇAISE DE RADIOLOGIE. Recommandations générales pour l'élaboration d'un compte-rendu radiologique (CRR). J Radiol 2007; 88:304-6.

9. INTERNATIONAL COMMISSION ON RADIOLOGICAL PROTECTION (ICRP). Las recomendaciones 2007 de la Comisión Internacional de Protección Radiológica. Publicación 103. Ann ICRP 2007; 37:1-117.

10. España. Real Decreto $1085 / 2009$, de 3 de julio, por el que se aprueba el reglamento sobre instalación y utilización de aparatos de rayos $\mathrm{X}$ con fines de diagnóstico médico [citado el 17 de mar. 2019]. https://www.boe.es/boe/dias/2009/07/18/pdfs/ BOE-A-2009-11932.pdf

11. Chile. Decreto Supremo $N^{\circ} 133$ del 23/08/1984 del Ministerio de Salud aprueba reglamento sobre autorizaciones para instalaciones radiactivas o equipos generadores de radiaciones ionizantes, personal que se desempeña en ellas u opere tales equipos y otras actividades afines [citado el 17 de mar. 2019]. https://www.leychile.cl/Navegar?idNorma $=9794$

12. EUROPEAN COUNCIL DIRECTIVE 2013/59/ Euratom laying down basic safety standards for protection against the dangers arising from exposure to ionising radiation, and repealing Directives 89/618/Euratom, 90/641/Euratom, 96/29/Euratom, 97/43/Euratom and 2003/122/Euratom. OJ of the EU 2014. L13; 57:1-73.

13. European Commission. Radiation protection No 172. Cone beam CT for dental and maxillofacial radiology: Evidence-based guidelines. 2012.

14. Carter L, Farman AG, Geist J, Scarfe WC, Angelopoulos C, Nair MK et al. American Academy of Oral and Maxillofacial Radiology executive opinion statement on performing and interpreting diagnostic cone beam computed tomography. Oral Surg Oral Med Oral Pathol Oral Radiol Endod 2008; 106:561-2.
15. Wilcox JR. The written radiology report. Appl Radiol 2006; 35:33-7.

16. Pinto A, Brunese L. Spectrum of diagnostic errors in radiology. World J Radiol 2010; 2:377-83.

17. Lozano KDS. Radiologists' ethical and professional obligations. Virtual Mentor 2007; 9:769-72.

18. INTERNATIONAL COMMISSION ON RADIOLOGICAL PROTECTION (ICRP). Ethical foundations of the system of radiological protection. Publication 138. Ann ICRP 2018; 47:1-56.

19. Mathioudakis A, Rousalova I, Gagnat AA, Saad N, Hardavella G. How to keep good clinical records. Breathe (Sheff) 2016; 12:371-5.

20. Armstrong 2nd JD. Morality, ethics, and radiologists' responsibilities. AJR Am J Roentgenol 1999; 173:279-84.

21. Edwards R, Altalibi M, Flores-Mir C. The frequency and nature of incidental findings in cone-beam computed tomographic scans of the head and neck region: a systematic review. J Am Dent Assoc 2013; 144:161-70.

22. American Dental Association and Food and Drug Administration. Dental radiographic examinations: recommendations for patient selection and limiting radiation exposure [citado el 17 de mar. 2019]. https://www.ada. org/ /media/ADA/Member\%20Center/FIles/ Dental_Radiographic_Examinations_2012.pdf

23. Depasquale $\mathrm{R}$, Crockford MP. Are radiology request forms adequately filled in? An audit assessing local practice. Malta Med J 2005; 17:36-8.

24. Metsälä E, Henner A, Ekholm M. Quality assurance in digital dental imaging: a systematic review. Acta Odontol Scand 2014; 72:362-71.

25. de las Heras Gala H, Torresin A, Dasu A, Rampado $\mathrm{O}$, Delis H, Hernández-Girón I et al. Quality control in cone-beam computed tomography (CBCT) EFOMP-ESTRO-IAEA protocol (summary report). Phys Med 2017; 39:67-72.

26. Moshfeghi M, Shahbazian M, Sajadi SS, Sajadi $\mathrm{S}$, Ansari H. Effects of different viewing conditions on radiographic interpretation. J Dent 
(Tehran) 2015; 12:853-8.

27. Zinman EJ, White SC, Tetradis S. Legal considerations in the use of cone beam computer tomography imaging. J Calif Dent Assoc 2010; 38:49-56.

28. Geist JR. The efficacy of diagnostic imaging should guide oral and maxillofacial radiology research. Oral Surg Oral Med Oral Pathol Oral Radiol 2017; 124:211-3.

29. Beacham JT, Geist JR, Yu Q, Himel VT, Sabey KA. Accuracy of cone-beam computed tomographic image interpretation by endodontists and endodontic residents. J Endod 2018; 44:571-5.

30. Srinivasa Babu A, Brooks ML. The malpractice liability of radiology reports: minimizing the risk. Radiographics 2015; 35:547-54.

31. Huda W, Abrahams RB. Radiographic techniques, contrast, and noise in x-ray imaging. AJR Am J Roentgenol 2015; 204:W126-31.

32. Schulze R, Heil U, Gross D, Bruellmann DD, Dranischnikow E, Schwanecke U et al. Artefacts in CBCT: a review. Dentomaxillofac Radiol 2011; 40:265-73.

33. Guerrero ME, Botetano R, Beltran J, Horner K, Jacobs $\mathrm{R}$. Can preoperative imaging help to predict postoperative outcome after wisdom tooth removal? A randomized controlled trial using panoramic radiography versus cone-beam CT. Clin Oral Investig 2014; 18:335-42.

34. Friedland B, Miles DA. Liabilities and risks of using cone beam computed tomography. Dent Clin North Am 2014; 58:671-85.

35. Singer SR, Creanga AG. Diagnostic imaging of malignant tumors in the orofacial region. Dent Clin North Am 2016; 60:143-65.

36. Goyal G, Padda S, Kaur B. Unusual incidental findings on intra- and extra-oral radiographs in North Indian population: a radiographic study. J Dent Allied Sci 2016; 5:74-8.

37. El Khateeb SM, Abu-Hammad O, Fadel H, Dar-Odeh N. A Retrospective analysis of radiographic jaw findings in young Women; prevalence and predictors. J Int Soc Prev Community Dent 2017; 7:22-7.
38. Syed AZ, Hawkins A, Alluri LS, Jadallah B, Shahid $\mathrm{K}$, Landers $M$ et al. Rare finding of Eustachian tube calcifications with cone-beam computed tomography. Imaging Sci Dent 2017; 47:275-9.

39. Devang Divakar D, Mavinapalla S, Al Kheraif AA, Ramakrishnaiah R, Abd Rahim MF, Khan AA. Incidental radiographic finding of the concealed art of susuk. Med Sci Law 2016; 56:230-2.

40. Adisen MZ, Okkesim A, Misirlioglu M. The importance of early diagnosis of Gardner's syndrome in dental examination. Niger J Clin Pract 2018; 21:114-6.

41. Abdalla-Aslan R, Friedlander-Barenboim S, Aframian DJ, Maly A, Nadler C. Ameloblastoma incidentally detected in cone-beam computed tomography sialography: a case report and review of the literature. J Am Dent Assoc 2018; 149:1073-80.

42. Gutmacher Z, Teich S, Lin S, Ghantous Y, Abu El-Naaj I. Practitioners' challenges in diagnosing oral and maxillofacial tumors. Quintessence Int 2016; 47:603-8.

43. IADMFR. International Association of Dento-Maxillo-Facial Radiology Newsletter. Dentomaxillofac Radiol 2018; 47:2.

44. Selim DG, Sexton C, Monsour P. Dentomaxillofacial radiology in Australia and dentist satisfaction with radiology reports. Aust Dent J 2018; 63:402-13.

45. IADMFR. International Association of Dento-Maxillo-Facial Radiology [citado el 17 de mar. 2019]. https://www.iadmfr.one/

46. Aps JKM. Flemish general dental practitioners' knowledge of dental radiology. Dentomaxillofac Radiol 2010; 39:113-8.

47. Ardakani FE, Sarayesh V. Knowledge of correct prescription of radiographs among dentists in Yazd, Iran. J Dent Res Dent Clin Dent Prospects 2008; 2:95-8.

48. Yakoumakis EN, Tierris CE, Stefanou EP, Phanourakis IG, Proukakis CC. Image quality assessment and radiation doses in intraoral radiography. Oral Surg Oral Med Oral Pathol Oral Radiol Endod 2001; 91:362-8. 
49. Estay J, Bersezio C, Arias R, Fernández E, Oliveira Junior OB, Ferrarezi de Andrade $M$ et al. Effect of clinical experience on accuracy and reliability of radiographic caries detection. Int $\mathrm{J}$ Odontostomat 2017; 11:347-52.

50. Pakbaznejad Esmaeili E, Pakkala T, Haukka J, Siukosaari P. Low reproducibility between oral radiologists and general dentists with regards to radiographic diagnosis of caries. Acta Odontol Scand 2018; 76:346-50.

51. Ahmed F, Brooks SL, Kapila SD. Efficacy of identifying maxillofacial lesions in cone-beam computed tomographs by orthodontists and orthodontic residents with third-party software. Am J Orthod Dentofacial Orthop 2012; 141:451-9.

52. Parker JM, Mol A, Rivera EM, Tawil PZ. Cone-beam computed tomography uses in clinical endodontics: observer variability in detecting periapical lesions. J Endod 2017; 43:184-7.

53. British Society for Dental and Maxillofacial Radiology. Curriculum for Specialist Training in Dental and Maxillofacial Radiology [citado el 17 de mar. 2019]. https://www.nwpgmd.nhs.uk/ sites/default/files/Dental\%20Maxillofacial\%20 Radiology.pdf

54. Bamgbose BO, Suwaid MA, Kaura MA, Sugianto I, Hisatomi M, Asaumi J. Current status of oral and maxillofacial radiology in West Africa. Oral Radiol 2018; 34:105-12.
55. Cha JY, Mah J, Sinclair P. Incidental findings in the maxillofacial area with 3-dimensional cone-beam imaging. Am J Orthod Dentofacial Orthop 2007; 132:7-14.

56. McGuigan MB, Duncan HF, Horner K. An analysis of effective dose optimization and its impact on image quality and diagnostic efficacy relating to dental cone beam computed tomography (CBCT). Swiss Dent J 2018; 128:297-316.

57. Omami G. Oral and maxillofacial radiology: the challenge of change. Imaging Sci Dent 2015; 45:267-8.

58. Dave M, Horner K. Challenges in X-ray diagnosis: a review of referrals for specialist opinion. Br Dent J 2017; 222:431-7.

59. Rai S, Misra D, Dhawan A, Tyagi K, Prabhat $M$, Khatri M. Knowledge, awareness, and aptitude of general dentists toward dental radiology and CBCT: a questionnaire study. J Indian Acad Oral Med Radiol 2018; 30:110-5.

\author{
AUTOR DE CORRESPONDENCIA: \\ Alejandro Hidalgo Rivas \\ Escuela de Odontología \\ Universidad de Talca \\ Avenida Lircay S/N \\ Talca, Chile \\ Teléfono +56-71-2200476 \\ Correo electrónico: ahidalgo@utalca.cl
}

